Vol. 76 (2007) [55-68]

\title{
A RANK FOR RIGHT CONGRUENCES ON INVERSE SEMIGROUPS
}

\author{
Victoria Gould
}

The $S$-rank (where 'S' abbreviates 'sandwich') of a right congruence $\rho$ on a semigroup $S$ is the Cantor-Bendixson rank of $\rho$ in the lattice of right congruences $\mathcal{R C}$ of $S$ with respect to a topology we call the finite type topology. If every $\rho \in \mathcal{R C}$ possesses $S$ rank, then $S$ is ranked. It is known that every right Noetherian semigroup is ranked and every ranked inverse semigroup is weakly right Noetherian. Moreover, if $S$ is ranked, then so is every maximal subgroup of $S$. We show that a Brandt semigroup $\mathcal{B}^{0}(G, I)$ is ranked if and only if $G$ is ranked and $I$ is finite.

We establish a correspondence between the lattice of congruences on a chain $E$, and the lattice of right congruences contained within the least group congruence on any inverse semigroup $S$ with semilattice of idempotents $E(S) \cong E$. Consequently we argue that the (inverse) bicyclic monoid $B$ is not ranked; moreover, a ranked semigroup cannot contain a bicyclic $\mathcal{J}$-class. On the other hand, $B$ is weakly right Noetherian, and possesses trivial (hence ranked) subgroups.

Our notion of rank arose from considering stability properties of the theory $T_{S}$ of existentially closed (right) $S$-sets over a right coherent monoid $S$. The property of right coherence guarantees that the existentially closed $S$-sets form an axiomatisable class. We argue that $B$ is right coherent. As a consequence, it follows from known results that $T_{B}$ is a theory of $B$-sets that is superstable but not totally transcendental.

\section{INTRODUCTION}

Suppose that $S$ is a semigroup: we denote by $\mathcal{R C}=\mathcal{R} \mathcal{C}_{S}$ the lattice of right congruences of $S$. The notion of $S$-rank for $\rho \in \mathcal{R C}$ was introduced in [7], in an attempt to find a property intermediate between that of being right Noetherian and of being weakly right Noetherian. Such a property was sought in order to solve an open problem in the model theory of $S$-sets over a monoid $S$ (see [7] and below for further details). Recall that a semigroup is right Noetherian if every right congruence is finitely generated, and weakly right Noetherian if every right ideal is finitely generated. By considering Rees right congruences, it is easy to see that a right Noetherian semigroup is weakly right Noetherian. The converse is, however, far from true: to see this the reader need only consider a group a having a subgroup lattice that does not have the ascending chain condition.

Received 13th Nobember, 2006

Copyright Clearance Centre, Inc. Serial-fee code: 0004-9727/07 \$A2.00+0.00. 
The $S$-rank of $\rho \in \mathcal{R C}$ is the Cantor-Bendixson rank of $\rho$ with respect to the finite type topology. The topology and the calculation of rank is given explicitly in Section 2 . For the purposes of this introduction, it is enough to say that the $S$-rank of $\rho \in \mathcal{R C}$ is an ordinal, or $\infty$ (considered to be greater than every ordinal), that measures how far $\rho$ is from being finitely generated and finitely covered in $\mathcal{R C}$. A semigroup is ranked if every $\rho \in \mathcal{R C}$ has $S$-rank strictly less than $\infty$. We showed in [7] that a right Noetherian semigroup is ranked, and a ranked inverse semigroup is weakly right Noetherian.

The aim of this paper is to consider ranked inverse semigroups. From [7], if a Brandt semigroup $\mathcal{B}^{0}(G, I)$ over a group $G$ is ranked, then $I$ is finite and $G$ is ranked. In Section 3 we prove the converse. Right congruences on Brandt semigroups may be described using inverse subsemigroups containing the 0 element [12]; for the purposes of our arguments it is easier to take a direct approach.

In Section 4 we concentrate on an E-unitary inverse semigroup $S$ having a chain $E(S)$ of idempotents. By analysing right congruences contained in the least group congruence $\sigma$, we can show that if $S$ is ranked then $E(S)$ is finite. Consequently, the bicyclic monoid $B$ is not ranked, even though it is weakly right Noetherian and has trivial (hence ranked) subgroups. It follows that a ranked semigroup cannot contain a bicyclic $\mathcal{J}$-class. Theorem 1.3 of [8] gives that the 0-simple principal factors of a right Noetherian semigroup are completely 0 -simple. We conjecture the same is true for ranked semigroups.

Our approach to rank was inspired by the model-theoretic Morley rank. From [7], every (complete 1)-type of the theory $T_{S}$ of existentially closed (right) $S$-sets over a right coherent monoid $S$ has Morley rank, that is, $T_{S}$ is totally transcendental, if and only if $S$ is weakly right Noetherian and ranked. From $[11,4] T_{S}$ is superstable if and only if $S$ is weakly right Noetherian. In Section 5 we show that $B$ is right coherent. Thus $T_{B}$ exists, is superstable, but not totally transcendental. For background in model theory we recommend [2] and [3]. The reader interested in stability theory can find further details in the books $[1,9,13]$.

We remark that $S$-rank was introduced in [7] for pairs $(I, \rho)$ where $\rho \in \mathcal{R C}$ and $I$ is a $\rho$-saturated right ideal of $S$. Our aim in doing so was to get a close bound on the Morley rank of types over the theory $T_{S}$, where $S$ is a right coherent monoid. However, if all pairs of the form $(\emptyset, \rho)$ possess $S$-rank then so do all pairs $(I, \rho)$. For the purposes of this paper, which makes no explicit model-theoretic considerations, it is enough to consider pairs $(\emptyset, \rho)$. Thus we define $S$-rank simply for elements of $\mathcal{R C}$.

\section{2. $S$-RANK FOR RIGHT CONGRUENCES}

Sandwich rank, or $S$-rank, for right congruences on a semigroup $S$ is defined as below. The terminology arose since we aim to 'sandwich' right congruences in intervals of the lattice $\mathcal{R C}$ determined by finite sets. Let $\nu$ denote a finitely generated right congruence 
on $S$ and let $K \subseteq S \times S$ be finite. Then we let

$$
[\nu, K]=\{\rho \in \mathcal{R C}: \nu \subseteq \rho \subseteq S \times S \backslash K\}
$$

and we say that $[\nu, K]$ is a subset of finite type. Clearly subsets of finite type are convex, where a subset $Y$ of a partially ordered set $X$ is convex if, for any $x, y, z \in X$, from $x \leqslant y \leqslant z$ and $x, z \in Y$, we deduce that $y \in Y$. Denoting the right congruence on $S$ generated by a subset $H$ of $S \times S$ by $\langle H\rangle$, it is easy to see that $[\mu, K] \cap[\nu, L]$ $=[\langle\mu \cup \nu\rangle, K \cup L] ;$ consequently, finite intersections of subsets of finite type are of finite type. Hence the set of subsets of finite type are a basis for a topology on $\mathcal{R C}$; we call this the finite type topology.

Let $\rho \in \mathcal{R C}$. The $S$-rank of $\rho, \mathrm{S}(\rho)$, is the Cantor-Bendixson rank of $\rho$ in $\mathcal{R C}$ equipped with the finite type topology. We make this explicit by defining subsets $\mathcal{R C}^{\alpha}$ of $\mathcal{R C}$ for each ordinal $\alpha$, as follows:

(I) $\mathcal{R C}^{0}=\mathcal{R C}$;

(II) if $\alpha$ is a limit ordinal, then

$$
\mathcal{R C}^{\alpha}=\cap\left\{\mathcal{R C}^{\beta}: \beta<\alpha\right\} ;
$$

(III) $\rho \in \mathcal{R C ^ { \alpha + 1 }}$ if and only if $\rho \in \mathcal{R \mathcal { C } ^ { \alpha }}$ and for each subset of finite type $[\nu, K]$ which contains $\rho$, there exists $\theta \in \mathcal{R C}$ such that

$$
\theta \in[\nu, K], \theta \neq \rho \text { and } \theta \in \mathcal{R C}^{\alpha} \text {. }
$$

The $S$-rank of $\rho \in \mathcal{R C}$ is $\mathrm{S}(\rho)$, where if $\rho \in \mathcal{R C ^ { \alpha }}$ for all $\alpha$ then $\mathrm{S}(\rho)=\infty$, and otherwise $\mathrm{S}(\rho)=\alpha$ where $\rho \in \mathcal{R C}^{\alpha} \backslash \mathcal{R C}^{\alpha+1}$. If $\mathrm{S}(\rho)<\infty$, then we say that $\rho$ has $S$-rank. Notice that for any $\rho \in \mathcal{R C}$ and ordinal $\alpha, \mathrm{S}(\rho) \geqslant \alpha$ if and only if $\rho \in \mathcal{R} \mathcal{C}^{\alpha}$. We remark that in $[7], S(\rho)$ is denoted by $S(\emptyset, \rho)$.

We say that a semigroup $S$ is ranked if every element of $\mathcal{R C}$ has $S$-rank. Notice that if $\rho$ is finitely generated and has finitely many covers $\left\{\rho_{i}: i \in I\right\}$, then choosing $\left(u_{i}, v_{i}\right) \in \rho_{i} \backslash \rho$, and putting

$$
K=\left\{\left(u_{i}, v_{i}\right): i \in I\right\},
$$

it is clear that $[\rho, K]$ isolates $\rho$, that is, $[\rho, K]=\{\rho\}$. Thus $\mathrm{S}(\rho)=0$. Hence any finite semigroup is ranked, and if the universal relation $\omega$ on any semigroup is finitely generated, then $S(\omega)=0$.

We recall the following results from [7], which will be needed in later sections.

Proposition 2.1. Let $S, T$ be semigroups and let $\psi: S \rightarrow T$ be an onto morphism. If $S$ is ranked, then so is $T$.

Proposition 2.2. Let $S$ be a semigroup and let $T$ be a monoid principal factor, a monoid $\mathcal{J}$-class, or a maximal subgroup of $S$. If $S$ is ranked, then so is $T$.

Proposition 2.3. Let $S$ be a ranked inverse semigroup. Then $S$ is weakly right Noetherian. 


\section{RANKED BRANDT SEMIGROUPS}

Let $\mathcal{B}^{0}=\mathcal{B}^{0}(G, I)$ be a Brandt semigroup. If $\mathcal{B}^{0}$ is ranked, then from Propositions 2.2 and 2.3 we know that $G$ is ranked and $I$ is finite. The aim of this section is to prove the converse.

To this end, let $\mathcal{B}^{0}=\mathcal{B}^{0}(G, I)$ be a Brandt semigroup. For each $i \in I$ we put

$$
G_{i}=\{(i, a, i): a \in G\},
$$

so that $G_{i}$ is a subgroup of $\mathcal{B}^{0}$ isomorphic to $G$. For any $\rho \in \mathcal{R C}=\mathcal{R C}_{B^{0}}$ we put

$$
\rho_{i}=\rho \cap\left(G_{i} \times G_{i}\right)
$$

so that $\rho_{i}$ is a right congruence on $G_{i}$. We also denote the $\rho$-class of 0 by $U_{\rho}$, so that in particular $U_{\rho}$ is a right ideal.

We make the observation that

$$
\omega=\langle\{(0,(i, 1, i)): i \in I\}\rangle .
$$

THEOREM 3.1. Suppose that $\mathcal{B}^{0}=\mathcal{B}^{0}(G, I)$ is a Brandt semigroup over a group $G$. Then $\mathcal{B}^{0}$ is ranked if and only if $G$ is ranked and $I$ is finite.

Proof: We assume that $G$ is ranked and $I$ is finite. From the observation preceding the theorem, $\omega$ is finitely generated. As remarked in Section 2 , it follows that $S(\omega)=0$.

Our aim then is to show that if $\rho \in \mathcal{R C}$ with

$$
\left\{i \in I:(i, 1, i) \notin U_{\rho}\right\} \neq \emptyset \text {, }
$$

then we have that $S(\rho)<\infty$.

Choose and fix a non-empty subset $J$ of $I$. Without loss of generality we can assume that

$$
J=\{1,2, \ldots, m\}
$$

for some $m \in \mathbb{N}$. Put $J^{\prime}=I \backslash J$ and

$$
U=\left\{(i, a, j): i \in J^{\prime}\right\} \cup\{0\},
$$

so that $U$ is a right ideal; if $J=I$, then $U=\{0\}$. Let

$$
\mathcal{R C}(J)=\left\{\rho \in \mathcal{R C}: U_{\rho}=U\right\}
$$

We proceed via a series of lemmas to show that every $\rho \in \mathcal{R C}(J)$ has $S$-rank.

LEMma 3.2. Let $\rho \in \mathcal{R C}(J)$. Then for any $i, j \in J$,

$$
(i, a, k) \rho(j, b, l) \text { implies that } k=l \text {. }
$$

Further,

$$
(i, a, k) \rho(j, b, k) \text { if and only if }(i, a, m) \rho(j, b, m)
$$

for any $m \in I$. 
Proof: If $(i, a, k) \rho(j, b, l)$, then as $\rho$ is a right congruence,

$$
(i, a, k)=(i, a, k)(k, 1, k) \rho(j, b, l)(k, 1, k)
$$

so that $k=l$ since $(i, a, k) \notin U$. Suppose now that

$$
(i, a, k) \rho(j, b, k)
$$

and $m \in I$. Then

$$
(i, a, k)(k, 1, m) \rho(j, b, k)(k, 1, m)
$$

so that

$$
(i, a, m) \rho(j, b, m)
$$

as required.

For any $\rho \in \mathcal{R C}(J)$ we define a relation $\tilde{\rho}$ on $J$ by the rule that $i \tilde{\rho} j$ if and only if there exist $(i, a, l),(j, b, l)$ with $(i, a, l) \rho(j, b, l)$. Clearly $\tilde{\rho}$ is reflexive and symmetric. If $i \tilde{\rho} j \tilde{\rho} k$ then, calling upon Lemma 3.2 , for some $(i, a, l),(j, b, l),(j, c, l),(k, d, l)$ we have that

$$
(i, a, l) \rho(j, b, l) \text { and }(j, c, l) \rho(k, d, l)
$$

so that

$$
(i, a, l) \rho(j, b, l)=(j, c, l)\left(l, c^{-1} b, l\right) \rho(k, d, l)\left(l, c^{-1} b, l\right)=\left(k, d c^{-1} b, l\right),
$$

thus $i \tilde{\rho} k$ and $\tilde{\rho}$ is an equivalence on $J$.

We denote by $|\rho|$ the number of $\tilde{\rho}$-classes, so that $1 \leqslant|\rho| \leqslant m$. If $\theta, \rho \in \mathcal{R C}(J)$ and $\rho \subseteq \theta$, then clearly $\tilde{\rho} \subseteq \tilde{\theta}$ so that $|\rho| \geqslant|\theta|$ and $|\rho|=|\theta|$ if and only if $\tilde{\rho}=\tilde{\theta}$.

For the meanwhile we fix $\rho \in \mathcal{R C}(J)$ and show how to construct a subset of finite type (of $\mathcal{R} \mathcal{C}_{B^{0}}$ ) containing $\rho$ from subsets of finite type (of $\mathcal{R C}_{G_{i}}$ ) containing $\rho_{i}, i \in J$. For distinct $i, j \in J$ with $i \tilde{\rho} j$ we have that

$$
(i, a, k) \rho(j, b, k)
$$

say, so that

$$
(i, 1, i) \rho\left(j, b a^{-1}, i\right) \text {. }
$$

We choose and fix $g_{i j} \in G$ with $(i, 1, i) \rho\left(j, g_{i j}, i\right)$. Suppose that for each $i \in J, \rho_{i} \in\left[\nu_{i}, K_{i}\right]$ where $\nu_{i}=\left\langle H_{i}\right\rangle$. We define $H, \nu$ and $K$ by

$$
\begin{gathered}
H=\bigcup_{i \in J} H_{i} \cup\left\{\left((i, 1, i),\left(j, g_{i j}, i\right)\right): i \neq j \in J, i \widetilde{\rho} j\right\} \cup\left\{((k, 1, k), 0): k \in J^{\prime}\right\}, \\
\nu=\langle H\rangle
\end{gathered}
$$

and

$$
K=\bigcup_{i \in J} K_{i} \cup\{((i, 1, i), 0): i \in J\} .
$$

It is easy to see that $\rho \in[\nu, K]$. Moreover, if $\theta \in[\nu, K]$, then $\theta \in \mathcal{R C}(J)$ and for each $j \in J, \theta_{i} \in\left[\nu_{i}, K_{i}\right]$. 
Lemma 3.3. Let $\rho, H, \nu$ and $K$ be as above. If $\theta \in[\nu, K]$ and $\theta_{i}=\rho_{i}$ for each $i \in J$, then $\rho \subseteq \theta$.

Proof: We know that $\theta \in \mathcal{R C}(J)$ so that in particular $U$ is the $\theta$-class of 0 and

$$
\theta \cap(U \times U)=U \times U=\rho \cap(U \times U) .
$$

Suppose now that $(i, a, k) \rho(j, b, k)$ for some $i, j \in J$. Then $(i, 1, i) \rho\left(j, b a^{-1}, i\right)$. If $i=j$ then $(i, 1, i) \rho_{i}\left(j, b a^{-1}, i\right)$; but $\rho_{i}=\theta_{i}$ so that $(i, 1, i) \theta\left(j, b a^{-1}, i\right)$, thus $(i, a, k) \theta(j, b, k)$.

Consider now the case where $i \neq j$. As $i \widetilde{\rho} j$ we have a pair $\left((i, 1, i),\left(j, g_{i j}, i\right)\right) \in H$. Hence

$$
\left(j, b a^{-1}, i\right) \rho\left(j, g_{i j}, i\right) .
$$

Lemma 3.2 and the fact that $\theta_{j}=\rho_{j}$ now yield

$$
\left(j, b a^{-1}, i\right) \theta\left(j, g_{i j}, i\right) .
$$

Since $H \subseteq \theta$ we have that

$$
(i, 1, i) \theta\left(j, b a^{-1}, i\right)
$$

so that

$$
(i, a, k) \theta(j, b, k)
$$

and $\rho \subseteq \theta$ as required.

LEMMA 3.4. Let $\rho, H, \nu$ and $K$ be as above. If $\theta \in[\nu, K], \theta_{i}=\rho_{i}$ for each $i \in J$ and $\widetilde{\theta}=\tilde{\rho}$, then $\theta=\rho$.

Proof: By Lemma 3.3, we know that $\rho \subseteq \theta$. For the opposite inclusion, suppose that $(i, a, k) \theta(j, b, k)$ for some $i, j \in J$. If $i \neq j$ then as $i \widetilde{\theta} j$ we must have that $i \widetilde{\rho} j$ so that there is a pair $\left((i, 1, i),\left(j, g_{i j}, i\right)\right) \in H$. Arguing as in the proof of Lemma 3.3 yields the result.

We can now proceed to complete our proof that if $\rho \in \mathcal{R C}(J)$, then $\rho$ has $S$-rank. We use induction on

$$
\left(\mathrm{S}\left(\rho_{1}\right), \mathrm{S}\left(\rho_{2}\right), \ldots, \mathrm{S}\left(\rho_{m}\right),|\rho|\right)
$$

where the product set has the lexicographic ordering.

Suppose first that $\mathrm{S}\left(\rho_{i}\right)=0$ for each $i \in J$ and $|\rho|=1$. Then each $\rho_{i}$ is isolated, say by $\left[\nu_{i}, K_{i}\right]$. Let $[\nu, K]$ be constructed as above, so that $\rho \in[\nu, K]$. If $\theta \in[\nu, K]$, then $\theta_{i} \in\left[\nu_{i}, K_{i}\right]$ so that as $\rho_{i}$ is isolated by this set, $\theta_{i}=\rho_{i}$ for each $i \in J$. By Lemma 3.3 we have that $\rho \subseteq \theta$. Hence

$$
1=|\rho| \geqslant|\theta|
$$

forces $|\rho|=|\theta|$ so that $\tilde{\rho}=\tilde{\theta}$. By Lemma 3.4 we have that $\rho=\theta$. Thus $[\nu, K]$ isolates $\rho$ and $\mathrm{S}(\rho)=0$. 
Proceeding by induction, we suppose that for all $\psi \in \mathcal{R C}(J)$ with

$$
\left(\mathrm{S}\left(\psi_{1}\right), \mathrm{S}\left(\psi_{2}\right), \ldots, \mathrm{S}\left(\psi_{m}\right),|\psi|\right)<\left(\mathrm{S}\left(\rho_{1}\right), \mathrm{S}\left(\rho_{2}\right), \ldots, \mathrm{S}\left(\rho_{m}\right),|\rho|\right)
$$

we have that $\mathrm{S}(\psi)<\infty$.

For each $i \in J$ let $\alpha_{i}=\mathrm{S}\left(\rho_{i}\right)$ and let $\left[\nu_{i}, K_{i}\right]$ isolate $\rho_{i}$ amongst right congruences of $G_{i}$ with $S$-rank greater than or equal to $\alpha_{i}$. Construct $[\nu, K]$ as above. If $\theta \in[\nu, K]$, then $\theta \in \mathcal{R C}(J)$ and $\theta_{i} \in\left[\nu_{i}, K_{i}\right]$. Consequently, $\mathrm{S}\left(\theta_{i}\right) \leqslant \mathrm{S}\left(\rho_{i}\right)$ for each $i$. If $\mathrm{S}\left(\theta_{i}\right)<\mathrm{S}\left(\rho_{i}\right)$ for some $i$, then

$$
\left(\mathrm{S}\left(\theta_{1}\right), \mathrm{S}\left(\theta_{2}\right), \ldots, \mathrm{S}\left(\theta_{m}\right),|\theta|\right)<\left(\mathrm{S}\left(\rho_{1}\right), \mathrm{S}\left(\rho_{2}\right), \ldots, \mathrm{S}\left(\rho_{m}\right),|\rho|\right)
$$

so that $\mathrm{S}(\theta)$ exists by our inductive assumption.

Otherwise, $\mathrm{S}\left(\theta_{i}\right)=\mathrm{S}\left(\rho_{i}\right)=\alpha_{i}$ for each $i$. But then $\theta_{i}=\rho_{i}$ for each $i$, so that by Lemma 3.3, $\rho \subseteq \theta$, giving that $|\rho| \geqslant|\theta|$. If this inequality is strict, then again we have that

$$
\left(\mathrm{S}\left(\theta_{1}\right), \mathrm{S}\left(\theta_{2}\right), \ldots, \mathrm{S}\left(\theta_{m}\right),|\theta|\right)<\left(\mathrm{S}\left(\rho_{1}\right), \mathrm{S}\left(\rho_{2}\right), \ldots, \mathrm{S}\left(\rho_{m}\right),|\rho|\right)
$$

and so $S(\theta)$ exists. On the other hand, if $|\rho|=|\theta|$, then $\tilde{\rho}=\tilde{\theta}$ so that $\rho=\theta$ by Lemma 3.4.

Our conclusion then is that for $\theta \in[\nu, K]$ we have that $\theta=\rho$ or $\mathrm{S}(\theta)<\infty$. Now if $\alpha$ is an ordinal larger than $\mathrm{S}(\theta)$ for any $\theta \in[\nu, K]$ with $\theta \neq \rho$ (and $\alpha$ certainly exists since $[\nu, K]$ is a set), we cannot have that $\mathrm{S}(\rho) \geqslant \alpha+1$. Hence $\mathrm{S}(\rho)<\infty$ as required.

\section{S-RANK FOR INVERSE SEMIGROUPS HAVING A CHAIN OF IDEMPOTENTS}

We recall that a semigroup $S$ is $E$-unitary if for any $e \in E(S)$ and $a \in S, e a \in E(S)$ implies that $a \in E(S)$. We say that $S$ is a chain semigroup if $E(S)$ forms a chain under its natural partial order. We prove in this section that if $S$ is a ranked E-unitary inverse chain semigroup, then $E(S)$ is finite. In view of Proposition 2.2, any ranked semigroup $S$ cannot contain an E-unitary inverse $\omega$-semigroup as a $\mathcal{J}$-class. In particular, it cannot contain a bicyclic $\mathcal{J}$-class.

Suppose that $S$ is an $E$-unitary inverse semigroup with chain of idempotents $E=E(S)$. We aim to utilise the results of [7] for ranked chains by describing certain elements of $\mathcal{R C}_{S}$ by their restriction in $\mathcal{R C _ { E }}$. We say that a partition

$$
\mathcal{E}=\left\{E_{i}: i \in I\right\}
$$

of $E$ is a convex partition if each $E_{i}$ is convex. Certainly such a partition inherits a partial ordering from $E$, so that $E_{i}<E_{j}$ if $x<y$ for all (any) $x \in E_{i}, y \in E_{j}$. It is easy to see that if $\rho \in \mathcal{R} \mathcal{C}_{E}$, then the $\rho$-classes form a convex partition. Conversely, the equivalence relation associated with a convex partition is a congruence on $E$. 
We recall that the least group congruence on an inverse semigroup $S$ is given by the rule

$$
a \sigma b \text { if and only if } e a=e b \text { for some } e \in E \text {. }
$$

Clearly, if $S$ is E-unitary, then $E$ forms a $\sigma$-class.

Suppose that $S$ is an inverse chain semigroup and let $\mathcal{E}$ be a convex partition of $E$. Let $\rho_{\mathcal{E}}$ be the relation on $S$ given by

$$
\rho_{\mathcal{E}}=\left\{(a, b): \exists i \in I \text { such that } a a^{\prime}, b b^{\prime} \in E_{i} \text { and } e a=e b \text { for some } e \in E_{i}\right\},
$$

where we use $s^{\prime}$ throughout to denote the inverse of an element $s \in S$.

LEMMA 4.1. Let $S$ be an inverse chain semigroup, and let $\mathcal{E}, \rho_{\mathcal{E}}$ be as above. Then $\rho_{\mathcal{E}}$ is a right congruence on $S$, contained in $\sigma$, which induces the partition $\mathcal{E}$ of $E$.

ProOF: It is clear that a convex partition satisfies condition $(N)$ of $[10]$. The leftright dual of $\left[10\right.$, Theorem 3.1] gives that $\rho_{\mathcal{E}}$ is a right congruence, indeed the minimum right congruence inducing the partition $\mathcal{E}$ of $E$. Clearly $\rho_{\mathcal{E}} \subseteq \sigma$.

The following technical result, which makes implicit use of the natural partial order on an inverse semigroup, will be used repeatedly.

LEMMA 4.2. Let $S$ be an E-unitary inverse chain semigroup, and let $a, b \in S$ with $a \sigma b$. Then $b a^{\prime}=a b^{\prime} \in E$. If $b \leqslant_{\mathcal{L}} a$ then $b a^{\prime}=b b^{\prime}$.

Proof: Since $a \sigma b$ we have that $e a=e b$ for some $e \in E$. Now

$$
e a a^{\prime}=e b a^{\prime}
$$

so that $b a^{\prime} \in E$ as $S$ is E-unitary. If $b \leqslant_{\mathcal{C}} a$ then $b=b a^{\prime} a$ so that

$$
b \leqslant_{\mathcal{R}} b a^{\prime} \leqslant_{\mathcal{R}} b
$$

and consequently, $b a^{\prime}=b b^{\prime}$ since $S$ is inverse.

For an inverse semigroup $S$ we let $\mathcal{G}$ denote the set of right congruences contained in $\sigma$.

LEMMA 4.3. Suppose that $S$ is an E-unitary inverse chain semigroup. Then for $\rho, \tau \in \mathcal{G}$,

$$
\rho \subseteq \tau \text { if and only if } \rho \cap(E \times E) \subseteq \tau \cap(E \times E) .
$$

ProOF: We suppose that $\rho \cap(E \times E) \subseteq \tau \cap(E \times E)$ and show that $\rho \subseteq \tau$.

Let $a, b \in S$ with $a \rho b$. Without loss of generality we assume that $b \leqslant_{\mathcal{L}} a$; Lemma 4.2 gives that $b a^{\prime}=b b^{\prime}$. Since $a a^{\prime} \rho b a^{\prime}$ and $\rho \cap(E \times E) \subseteq \tau \cap(E \times E)$ we must have that $a a^{\prime} \tau b a^{\prime}$. Hence, as $\tau$ is a right congruence,

$$
a=a a^{\prime} a \tau b a^{\prime} a=b .
$$


COROLlary 4.4. Suppose that $S$ is an E-unitary inverse chain semigroup. Then there is a bijection between $\mathcal{G}$ and the set of convex partitions of $E$.

Proof: If $\tau \in \mathcal{G}$ and $\mathcal{E}$ is the partition of $E$ induced by $\tau$, then as commented earlier, $\mathcal{E}$ must be convex. On the other hand for any convex partition $\mathcal{F}$ of $E, \rho_{\mathcal{F}}$ induces $\mathcal{F}$. Invoking Lemma 4.3 gives our result.

Since the set of convex partitions of $E$ is in bijective correspondence with $\mathcal{R C} \mathcal{C}_{E}$, it follows that there is a bijection between $\mathcal{G}$ and $\mathcal{R C}$.

We proceed to examine sets of generators for right congruences in $\mathcal{G}$.

LEMMA 4.5. Let $S$ be an E-unitary inverse chain semigroup.

For any $\rho \in \mathcal{G}$, if

$$
\rho=\left\langle\left\{\left(a_{j}, b_{j}\right): j \in J\right\}\right\rangle
$$

then

$$
\rho=\left\langle\left\{\left(a_{j} a_{j}^{\prime}, b_{j} b_{j}^{\prime}\right): j \in J\right\}\right\rangle .
$$

Proof: Let $\rho \in \mathcal{G}$ with

$$
\rho=\left\langle\left\{\left(a_{j}, b_{j}\right): j \in J\right\}\right\rangle .
$$

Let $j \in J$ and assume that $b_{j} \leqslant c a_{j}$. By Lemma 4.2 we have that $b_{j} b_{j}^{\prime}=b_{j} a_{j}^{\prime}$ and

$$
a_{j} a_{j}^{\prime} \rho b_{j} a_{j}^{\prime}=b_{j} b_{j}^{\prime} \text {. }
$$

Hence

$$
\tau=\left\langle\left\{\left(a_{j} a_{j}^{\prime}, b_{j} b_{j}^{\prime}\right): j \in J\right\}\right\rangle \subseteq \rho
$$

Conversely,

$$
b_{j}=b_{j} a_{j}^{\prime} a_{j}=b_{j} b_{j}^{\prime} a_{j} \tau a_{j} a_{j}^{\prime} a_{j}=a_{j},
$$

so that $\rho \subseteq \tau$ and we have that $\rho=\tau$.

For the purposes of the next result, we denote the right congruence on a subsemigroup $T$ of $S$ generated by $H \subseteq T \times T$ as $\langle H\rangle_{T}$.

LEMMA 4.6. Let $S$ be an $E$-unitary inverse chain semigroup, let $H \subseteq E \times E$ and let $\rho=\langle H\rangle_{S}$. Then $\rho \in \mathcal{G}$ and $\rho \cap(E \times E)=\langle H\rangle_{E}$.

Proof: Let $H \subseteq E \times E$. If $a \rho b$, then $a=b$ (so that $a \sigma b$ ), or there is a sequence

$$
a=c_{1} t_{1}, d_{1} t_{1}=c_{2} t_{2}, \ldots, d_{l} t_{l}=b,
$$

where for each $k,\left(c_{k}, d_{k}\right)$ or $\left(d_{k}, c_{k}\right)$ belongs to $H$ and $t_{k} \in S^{1}$. Since $E$ is a chain we can find a least element $e$ in

$$
\left\{c_{1}, d_{1}, \ldots, c_{l}, d_{l}\right\}
$$

and then

$$
e a=e c_{1} t_{1}=e t_{1}=e d_{1} t_{1}=e c_{2} t_{2}=\ldots=e d_{l} t_{l}=e b
$$


so that $a \sigma b$ and $\rho \in \mathcal{G}$.

Clearly

$$
\langle H\rangle_{E} \subseteq \rho \cap(E \times E) .
$$

On the other hand, if $e, f \in E$ and $e \rho f$, then $e=f$ or there is a sequence

$$
e=u_{1} s_{1}, v_{1} s_{1}=u_{2} s_{2}, \ldots, v_{l} s_{l}=f
$$

where for each $k,\left(u_{k}, v_{k}\right)$ or $\left(v_{k}, u_{k}\right)$ is in $H$ and $s_{k} \in S^{1}$. Since $S$ is inverse we have a sequence

$$
e=u_{1} s_{1} s_{1}^{\prime}, v_{1} s_{1} s_{1}^{\prime}=u_{2} s_{2} s_{2}^{\prime}, \ldots, v_{l} s_{l} s_{l}^{\prime}=f,
$$

so that $e\langle H\rangle_{E} f$. Thus

$$
\rho \cap(E \times E)=\langle H\rangle_{E}
$$

Proposition 4.7. Suppose that $S$ is an E-unitary inverse chain semigroup and let $\rho \in \mathcal{G}$. Then $\rho$ is finitely generated (in $\mathcal{R C}_{S}$ ) if and only if $\rho \cap(E \times E)$ is finitely generated (in $\mathcal{R C}_{E}$ ).

Proof: If $\rho$ is finitely generated, then by Lemma $4.5, \rho$ has a finite set of generators $H$ where $H \subseteq E \times E$. By Lemma 4.6,

$$
\rho \cap(E \times E)=\langle H\rangle_{E}
$$

and thus is finitely generated.

Conversely, suppose that $\rho \cap(E \times E)$ is finitely generated by $F$. If $a \rho b$, then by Lemma 4.5, $a a^{\prime} \rho b b^{\prime}$ so that $a a^{\prime}\langle F\rangle_{E} b b^{\prime}$. Hence $a a^{\prime}=b b^{\prime}$ or there is a sequence

$$
a a^{\prime}=c_{1} t_{1}, d_{1} t_{1}=c_{2} t_{2}, \ldots, d_{l} t_{l}=b b^{\prime},
$$

where for each $k,\left(c_{k}, d_{k}\right)$ or $\left(d_{k}, c_{k}\right) \in F$ and $t_{k} \in E^{1}$. Without loss of generality, assume that $b \leqslant_{\mathcal{L}} a$ so that $b b^{\prime}=b a^{\prime}$. If $a a^{\prime}=b b^{\prime}$ then

$$
a=a a^{\prime} a=b b^{\prime} a=b a^{\prime} a=b .
$$

Otherwise,

$$
a=a a^{\prime} a=c_{1} t_{1} a, d_{1} t_{1} a=c_{2} t_{2} a, \ldots, d_{1} t_{l} a=b b^{\prime} a=b a^{\prime} a=b,
$$

so that $a\langle F\rangle_{S} b$. Consequently, $\rho=\langle F\rangle_{S}$ and hence is finitely generated.

We are finally in a position to prove the main result of this section. To do so we call upon the corresponding result in [7] for the case of $S$ a chain.

THEOREM 4.8. Suppose that $S$ is an E-unitary inverse chain semigroup. If $S$ is ranked, then $E$ is finite. 
ProOF: We proceed by transfinite induction to show that for any $\rho \in \mathcal{G}$ that is not finitely generated, and for any ordinal $\alpha, \mathrm{S}(\rho) \geqslant \alpha$. As usual, the step at limit ordinals is trivial. Suppose now that $\mathrm{S}(\mu) \geqslant \alpha$ for all $\mu \in \mathcal{G}$ that are not finitely generated. Let $\rho \in \mathcal{G}$ where $\rho$ is not finitely generated and suppose that

$$
\rho \in[\nu, K]
$$

where $[\nu, K]$ is a subset of finite type. Clearly

$$
\nu \subset \rho \subseteq \sigma .
$$

By Proposition 4.7, $\nu \cap(E \times E)$ is finitely generated but $\rho \cap(E \times E)$ is not. From [7, Lemma 5.6], we can find a non-finitely generated congruence $\theta$ on $E$ such that

$$
\nu \cap(E \times E) \subset \theta \subset \rho \cap(E \times E) .
$$

Let $\psi=\langle\theta\rangle_{S}$ so that $\psi \in \mathcal{G}$ from Lemma 4.6 and

$$
\psi \cap(E \times E)=\langle\theta\rangle_{E}=\theta
$$

From Proposition 4.7 we have that $\psi$ is not finitely generated. Lemma 4.3 gives that

$$
\nu \subset \psi \subset \rho .
$$

Thus $\psi \in[\nu, K]$ and $\psi \neq \rho$. Our inductive hypothesis tells us that $\mathrm{S}(\psi) \geqslant \alpha$, so that $\mathrm{S}(\rho) \geqslant \alpha+1$.

It follows that for any non-finitely generated $\rho \in \mathcal{G}, \mathrm{S}(\rho)=\infty$. If $E$ is infinite, then it contains an unbounded subset, so that as in [7, Proposition 5.7], $\mathcal{R \mathcal { C } _ { E }}$ contains a non-finitely generated congruence $\theta$. Then $\langle\theta\rangle_{S}$ fails to be finitely generated, so that $\mathrm{S}\left(\langle\theta\rangle_{S}\right)=\infty$ and $S$ is not ranked.

Corollary 4.9. Suppose that $S$ is a ranked semigroup. Then $S$ cannot contain a $\mathcal{J}$-class that is an inverse $\omega$-monoid. In particular, $S$ cannot contain a $\mathcal{J}$-class isomorphic to $B$.

Proof: Let $S$ be ranked. If $S$ contains an inverse $\omega$-semigroup $T$ as a $\mathcal{J}$-class, then $T$ is perforce ranked by Proposition 2.2. As $T$ is a $\mathcal{J}$-class of $S, T$ is a simple inverse $\omega$-semigroup: it need not be E-unitary but, from the characterisation of $T$ as a BruckReilly semigroup, it is clear that there is an onto morphism from $T$ to the bicyclic monoid $B$. Thus by Proposition 2.1, $B$ is ranked. But $B$ is an E-unitary inverse $\omega$-semigroup, so cannot be ranked in view of Theorem 4.8. 


\section{THE BICYCLIC MONOID IS RIGHT COHERENT}

A monoid $S$ is right coherent if every finitely generated $S$-subset of any finitely presented (right) $S$-set is finitely presented. It is known that $S$ is right coherent if and only if the theory of $S$-sets has a model companion; the model companion is then a set of sentences which axiomatises the existentially closed $S$-sets. Further details can be found in $[5,14]$. In [6] we show that an inverse monoid with central idempotents is right coherent. The aim of this section is to extend this result to show that an inverse monoid with an inversely well-ordered chain of idempotents is right coherent.

Lemma 5.1. Let $I$ be a right ideal of a monoid $S$, let $\rho \in \mathcal{R C}$ and let $x, y \in S$.

(i) The set

$$
I \rho=\{s \in S: s \rho a \text { for some } a \in I\}=\cup\{[a]: a \in I\}
$$

is a right ideal of $S$ containing $I$.

(ii) The set

$$
(I, x)=\{t \in S: x t \in I\}
$$

is empty or is a right ideal.

(iii) If $x \rho y$ then

$$
(I \rho, x)=(I \rho, y) .
$$

Proof: It is easy to see that (i) and (ii) hold. For (iii), observe that $x t \in I \rho$ if and only if $y t \in I \rho$, since $I \rho$ is a union of $\rho$-classes.

THEOREM 5.2. Let $S$ be a regular monoid such that every right ideal is principal. Then $S$ is right coherent.

Proof: Suppose that $\rho$ is a finitely generated right congruence on $S$. Since every right ideal of $S$ is principal, the set of right ideals of $S$ is linearly ordered by inclusion. Clearly then for $[a],[b] \in S / \rho$, we have that $[a] S \cap[b] S$ equals $[a] S$ or $[b] S$. From $[6$, Corollary 3.4], it remains to show that the right congruence

$$
r([a])=\{(u, v) \in S \times S: a u \rho a v\}
$$

is finitely generated.

Let $\left\{\left(x_{i}, y_{i}\right)\right\}$ be a finite set of generators for $\rho$ and let $[a] \in S / \rho$. Then $(a S) \rho$ is a right ideal of $S$, so by assumption

$$
\text { (aS) } \rho=e S \text { for some } e \in E .
$$

Clearly $e a=a$. Now $e \in(a S) \rho$ gives that $e \rho a t$ for some $t \in S$. Consequently,

$$
a=e a \rho a t a
$$


so that

$$
(1, t a) \in r([a])
$$

For any $i$,

$$
\left(e S, x_{i}\right)=\left(e S, y_{i}\right)=\emptyset
$$

or

$$
\left(e S, x_{i}\right)=\left(e S, y_{i}\right)=g_{i} S \text { for some } g_{i} \in E(S),
$$

using Lemma 5.1. In the latter case,

$$
a t x_{i} g_{i} \rho e x_{i} g_{i}=x_{i} g_{i} \rho y_{i} g_{i}=e y_{i} g_{i} \rho a t y_{i} g_{i}
$$

so that

$$
\left(t x_{i} g_{i}, t y_{i} g_{i}\right) \in r([a])
$$

Putting

$$
\tau=\left\langle\left\{(1, t a),\left(t x_{i} g_{i}, t y_{i} g_{i}\right)\right\}\right\rangle
$$

we have shown that

$$
\tau \subseteq r([a]) .
$$

Conversely, suppose that $(u, v) \in r([a])$, so that au $\rho a v$. If $a u=a v$, then

$$
u \tau \operatorname{tau}=\operatorname{tav} \tau v
$$

Otherwise, we have a sequence

$$
a u=c_{1} t_{1}, d_{1} t_{1}=c_{2} t_{2}, \ldots, d_{l} t_{l}=a v,
$$

where for each $j \in\{1, \ldots, l\}, t_{j} \in S$ and

$$
\left(c_{j}, d_{j}\right)=\left(x_{i_{j}}, y_{i_{j}}\right) \text { or }\left(c_{j}, d_{j}\right)=\left(y_{i_{j}}, x_{i_{j}}\right)
$$

Observe that for each $j$,

$$
t_{j} \in\left(e S, x_{i_{j}}\right)=\left(e S, y_{i_{j}}\right)=g_{i_{j}} S
$$

so that

$$
t_{j}=g_{i_{j}} t_{j}
$$

We rewrite our sequence as

$$
a u=c_{1} g_{i_{1}} t_{1}, d_{1} g_{i_{1}} t_{1}=c_{2} g_{i_{2}} t_{2}, \ldots, d_{l} g_{i_{l}} t_{l}=a v,
$$

and multiply every equation by $t$ to obtain

$$
t a u=t c_{1} g_{i_{1}} t_{1}, t d_{1} g_{i_{1}} t_{1}=t c_{2} g_{i_{2}} t_{2}, \ldots, t d_{l} g_{i_{l}} t_{l}=t a v .
$$


Since for each $j$ we have that

$$
\left(t c_{j} g_{i_{j}}, t d_{j} g_{i_{j}}\right) \text { or }\left(t d_{j} g_{i_{j}}, t c_{j} g_{i_{j}}\right)
$$

is a generator of $\tau$, we obtain

$$
u \tau \operatorname{tau} \tau \operatorname{tav} \tau v
$$

so that

$$
r([a]) \subseteq \tau
$$

and we deduce that $r([a])=\tau$ is finitely generated as required.

COROLLARY 5.3. The bicyclic monoid is right coherent.

\section{REFERENCES}

[1] J.T. Baldwin, Fundamentals of stability theory (Springer-Verlag, Berlin, 1988).

[2] C.C. Chang and H.K. Keisler, Model theory (North-Holland, Amsterdam, 1973).

[3] H.B. Enderton, A mathematical introduction to logic (Academic Press, New York, 1972).

[4] J.B. Fountain and V.A.R Gould, 'Stability of the theory of existentially closed $S$-sets over a right coherent monoid $S$ ', (preprint), http://www-users.york.ac.uk/ varg1/gpubs.htm.

[5] V.A.R. Gould, 'Model companions of $S$-systems', Quart. J. Math. Oxford 38 (1987), 189-211.

[6] V.A.R. Gould, 'Coherent monoids', J. Austral. Math. Soc. Ser. A 53 (1992), 166-182.

[7] V.A.R. Gould, 'A notion of rank for right congruences on semigroups', Comm. Algebro 33 (2005), 4631-4656.

[8] E. Hotzel, 'On semigroups with maximal conditions', Semigroup Forum 11 (1975/6), 337-362.

[9] D. Lascar, Stability in model theory (Longman, London, 1987).

[10] J. Meakin, 'One-sided congruences on inverse semigroups', Trans. Amer. Math. Soc. 206 (1975), 67-82.

[11] T.G. Mustafin, 'Stability of the theory of polygons', (in Russian), Trudy. Inst. Math (Novosibirisk) 8 (1988), 92-108. Translated in 'Model theory and applications' Amer. Math. Soc. Transl. 295 (1999) 205-223 .

[12] M. Petrich and S. Rankin, 'Right congruences on a Brandt semigroup', Soochow J. Math. 22 (1986), 85-106.

[13] A. Pillay, An introduction to stability theory (Oxford University Press, New York, 1983).

[14] W.H. Wheeler, 'Model companions and definability in existentially complete structures', Israel J. Math. 25 (1976), 305-330.

Department of Mathematics

University of York

Heslington, York YO10 5DD

United Kingdom

e-mail: varg1@york.ac.uk 